\title{
Lens epithelial cell regression on the posterior capsule with different intraocular lens materials
}

\author{
Emma J Hollick, David J Spalton, Paul G Ursell, Milind V Pande
}

\begin{abstract}
Backgroundlaims-Posterior capsular opacification (PCO) is caused by proliferation and migration of lens epithelial cells (LECs) across the posterior capsule and is the commonest cause of reduced vision after cataract surgery. The influence of intraocular lens (IOL) material on the process of LEC migration was studied. Methods-90 eyes underwent standardised extracapsular surgery, with capsulorhexis and "in the bag" IOL placement. They were randomised to receive a three piece $6 \mathrm{~mm}$ lens of PMMA, silicone, or polyacrylic (AcrySof, Alcon, Fort Worth, TX, USA). On days $7,30,90,180$, and years 1 and 2 high resolution digitised retroillumination images were taken of the posterior capsule. The presence of LECs was determined at 90 days and 2 years, and their progression or regression was established by serial examination of images.

Results-LECs were seen in $93 \%$ of silicone and $97 \%$ of PMMA IOLs at 90 days, compared with $46 \%$ of polyacrylic $(p<0.001)$. At year 2 LECs were present in all patients with silicone or PMMA lenses, whereas $62 \%$ of patients with polyacrylic IOLs had LECs $(p<0.001)$. Of those patients with LECs at day 90 LEC regression occurred in $8 \%$ with silicone IOLs and $15 \%$ of PMMA cases, compared with $83 \%$ of patients with polyacrylic IOLs $(\mathrm{p}<0.0001)$.

Conclusion-The presence of LECs on the posterior capsule was considerably lower with polyacrylic than PMMA or silicone IOLs and LEC regression occurred more frequently. The lower incidence of LECs and the higher rate of regression may explain why PCO formation appears to be reduced with polyacrylic lenses. This has important clinical implications for the prevention of PCO.

(Br F Ophthalmol 1998;82:1182-1188)
\end{abstract}

Posterior capsular opacification (PCO) is the commonest complication of cataract surgery with an incidence of between $10 \%$ and $50 \%$ by 2 years postoperatively. ${ }^{1}$ Residual lens epithelial cells (LECs), which remain in the capsular bag after cataract surgery, proliferate and transform. Opacification of the posterior capsule results from their migration and proliferation to produce Elschnig's pearls or fibroblastic transformation causing capsular fibrosis. ${ }^{2-4}$ In the peripheral capsular bag these processes cause no symptoms, but when cells encroach onto the visual axis they cause light scatter and visual deterioration.

PCO has important medical, social, and economic adverse effects and consequently there is considerable interest in its prevention. Biconvex and plano convex polymethyl methacrylate (PMMA) intraocular lenses (IOLs), ${ }^{5-7}$ as well as silicone plate haptic IOLs, ${ }^{8}$ have been reported to have a beneficial effect on PCO. However, other modifications of IOL design or surgical technique have had little influence on PCO rates. ${ }^{9}$ Attention has therefore focused on pharmacological agents to reduce the number of residual LECs left at the end of a cataract operation, ${ }^{10} 11$ to inhibit the proliferation of LECs after surgery, ${ }^{12}{ }^{13}$ or to alter their migration into the visual axis. ${ }^{14}$ Evidence of their efficacy comes largely from cell culture or animal studies, and their role in human cataract surgery remains unknown.

We have developed an imaging system that produces high resolution digitised retroillumination images of the posterior capsule ${ }^{15}$ and report for the first time that LEC behaviour is differentially influenced by the IOL material.

\section{Methods}

SURGICAL TECHNIQUE

Patients were recruited in a continuous cohort following approval from the hospital ethics committee. All surgery was performed between September 1993 and July 1994 by a single surgeon. Tight inclusion criteria were used to define the presence of senile cataract in an otherwise normal eye in patients over 55 years of age. Exclusion criteria were a history of previous intraocular surgery or laser treatment, diabetes mellitus requiring medical control, glaucoma, previous uveitis, or any posterior segment pathology precluding a postoperative vision of $20 / 40$ or better. Patients using topical medications (apart from lubricants) and any patients taking systemic steroids were excluded, in addition to those who had contralateral cataract surgery in the previous 4 months. All patients were assessed preoperatively by the same person.
Accepted for publication 11March 1998 
Table 1 Presence of lens epithelial calls (LECs) on the posterior capsule at days 90 and 720

\begin{tabular}{|c|c|c|}
\hline IOL type & $\begin{array}{l}\text { No of patients with } \\
\text { no LECs }\end{array}$ & $\begin{array}{l}\text { No of patients with } \\
\text { LECs }\end{array}$ \\
\hline \multicolumn{3}{|l|}{ PMMA } \\
\hline day $90, n=28$ & $1(4 \%)$ & $27(96 \%)$ \\
\hline year $2, n=25$ & $0(0 \%)$ & $25(100 \%)$ \\
\hline \multicolumn{3}{|l|}{ Silicone } \\
\hline day $90, \mathrm{n}=27$ & $2(7 \%)$ & $25(93 \%)$ \\
\hline year $2, n=21$ & $0(0 \%)$ & $21(100 \%)$ \\
\hline \multicolumn{3}{|l|}{ Polyacrylic } \\
\hline day $90, n=26$ & $14(54 \%)$ & $12(46 \%)$ \\
\hline year $2, n=16$ & $6(38 \%)$ & $10(62 \%)$ \\
\hline
\end{tabular}

Surgical technique and medication were standardised. Extracapsular cataract extraction with continuous curvilinear capsulorhexis was performed by a single surgeon using peribulbar anaesthesia. ${ }^{16}$ Preoperatively the pupil was dilated. A $10 \mathrm{~mm}$ superior corneal section was made and the anterior chamber was reformed with Healon (Pharmacia). The nucleus was hydroexpressed through a 5.5-6.5 $\mathrm{mm}$ capsulorhexis using balanced salt solution (BSS). Soft lens material was removed by manual irrigation aspiration with BSS; no attempt was made to reduce LECs by polishing the anterior capsule. The bag was reformed with Healon and the IOL inserted. Patients were randomised to receive one of three IOLs. These all had PMMA haptics with $6 \mathrm{~mm}$ optics of PMMA (Alcon MC60BM), silicone (Iolab L141U), or polyacrylic (AcrySof, Alcon MA60BM, Fort Worth, TX, USA). AcrySof is a proprietary copolymer of phenylethyl acrylate and phenylethyl methacrylate cross linked with 1.4 butanediol diacrylate. The angle of flexion of the haptics was 10 degrees with the PMMA and polyacrylic lenses and 5 degrees for silicone. The corneal wound was sutured with a continuous bootlace suture of 10/0 nylon and the Healon was thoroughly removed by irrigation with BSS. Subconjunctival cefuroxime $125 \mathrm{mg}$ was given at the end of the operation. Any surgical complications such as capsulorhexis rim tear, zonular dehiscence, failure to place the IOL into the capsular bag, posterior capsular rupture, or vitreous loss led to patient exclusion. Postoperatively all patients used Maxitrol, drops (neomycin, polymyxin, and dexamethasone $0.1 \%$ ) four times a day for 1 month. No non-steroidal anti-inflammatory preparation was used pre-, peri-, or postoperatively.

LENS EPITHELIAL CELL ASSESSMENT

Patients had standardised digital retroillumination imaging of the posterior capsule through dilated pupils at days $7,30,90,180$, and years 1 and 2 postoperatively, using a purpose built digitised retroillumination camera system. LECs can be easily visualised on the posterior capsule by 90 days. Their presence or absence on the posterior capsule was determined at day 90 and 2 years postoperatively for each IOL type (disregarding their extent). In those patients where they were present the changes in the migration and growth of these cells was determined by analysing consecutive images,
Table 2 Influence of IOL material on LEC growth in patients with LECs at 90 days

\begin{tabular}{llll}
\hline & LEC regress & LEC stable & LEC progress \\
\hline PMMA, $\mathrm{n}=27$ & $4(15 \%)$ & $1(4 \%)$ & $22(81 \%)$ \\
Silicone, $\mathrm{n}=25$ & $2(8 \%)$ & $2(8 \%)$ & $21(84 \%)$ \\
Polyacrylic, $\mathrm{n}=12$ & $10(83 \%)$ & $2(17 \%)$ & $0(0 \%)$ \\
\hline
\end{tabular}

comparing one image with the next, so that three observations made for each patient comparing changes between 90 and 180 days, 180 days and 1 year, and 1-2 years. LECs were determined to have progressed, be stable, or have regressed, between each image and the next in the chronological order. Any patient who missed the 90 day appointment or more than two follow up images was excluded.

In addition, the relation of the anterior capsulorhexis margin to the IOL optic at 90 days postoperatively was assessed and graded as either completely on the IOL optic for 360 degrees, partly on, or completely off the optic for 360 degrees. This was correlated with the presence of LECs on the posterior capsule at 90 days and, if LECs were present, we assessed whether the degree of capsulorhexis-optic contact had any influence on their progression, regression or stability.

\section{STATISTICS}

The difference between the three lens groups was analysed using a $\chi^{2}$ test.

\section{Results}

Ninety eyes of 81 patients were enrolled and allocated randomly by computer code to each lens group of 30 . The average age was 73 years, with a range of 55-89 years. There was no difference in age and sex distribution between the three groups.

The number of patients with images available for analysis at day 90 was 28 in the PMMA group, 27 for silicone, and 26 for polyacrylic. At 2 years 25 patients were available in the PMMA group, 21 for silicone, and 16 for polyacrylic.

PRESENCE OF LENS EPITHELIAL CELLS

Table 1 shows that in the PMMA group 96\% of patients had LECs present on the posterior capsule at 90 days, $93 \%$ with silicone, and $46 \%$ with polyacrylic. At 2 years all patients with PMMA and silicone IOLs had LECs on their posterior capsule, whereas $62 \%$ with polyacrylic had LECs on their capsules. Patients with polyacrylic lenses were significantly less likely to have LECs on their posterior capsules than those with PMMA or silicone at both day $90(\mathrm{p}<0.001)$ and 2 years $(\mathrm{p}<0.001)$.

FATE OF LENS EPITHELIAL CELLS

If LECs were present at day 90 their fate was assessed to determine whether they progressed, remained stable, or regressed (Table 2). In $81 \%$ of these patients with PMMA IOLs the LEC growth progressed between day 90 and 2 years (Figs $1 \mathrm{~A}, \mathrm{~B}, \mathrm{C}$ ), in $4 \%$ LEC growth was stable, and in $15 \%$ there was minor regression on some areas of the 

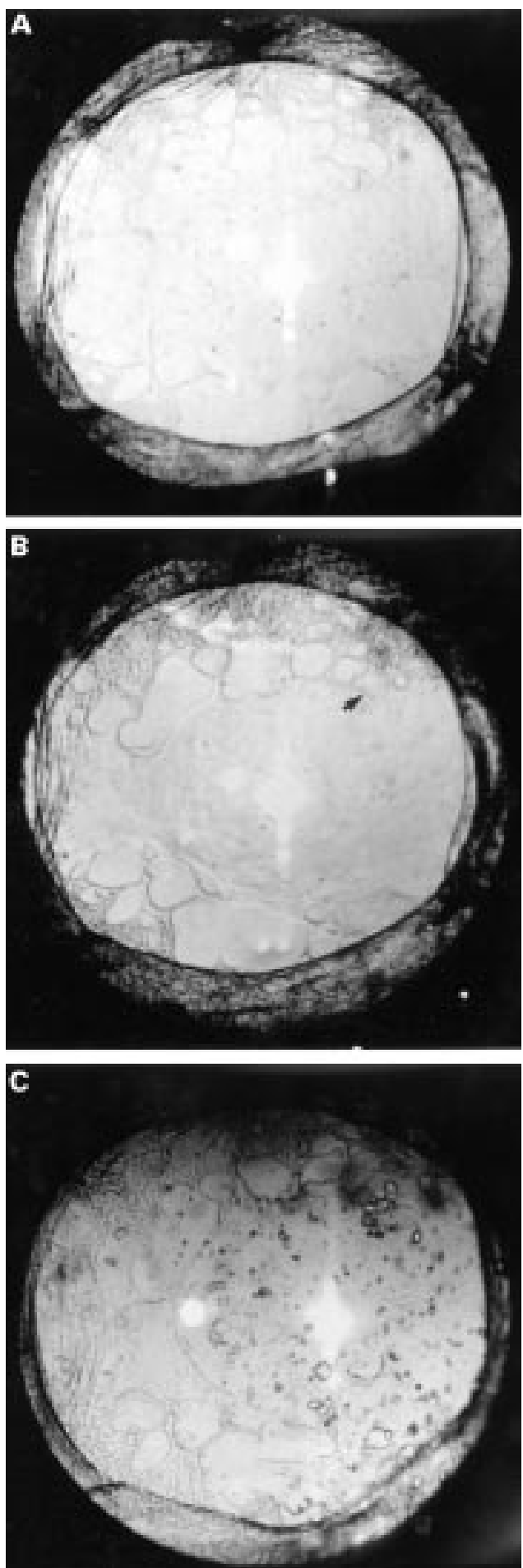

Figure 1 Lens epithelial cell (LEC) progression with a PMMA lens photographed at 3 months, 6 months, and 2 years postoperatively. (A) Three months after surgery the capsulorhexis lies on the anterior implant surface. A fine LEC membrane is seen on the posterior surface containing acellular spaces. (B) At 6 months the LEC membrane is thicker. The acellular spaces are more noticeable and, in some areas, have started to fill in (arrow). (C) At 2 years postoperatively the membrane is thicker with Elschnig pearl formation. Outlines of some of the previously acellular areas can still be made out.

capsule (Figs 2A, B, C). In patients with silicone IOLs and LECs at 90 days LEC progression was seen in $84 \%$ (Figs 3A, B, C), with $8 \%$ staying stable, and $8 \%$ showing evidence of regression (Figs 4A, B, C). There
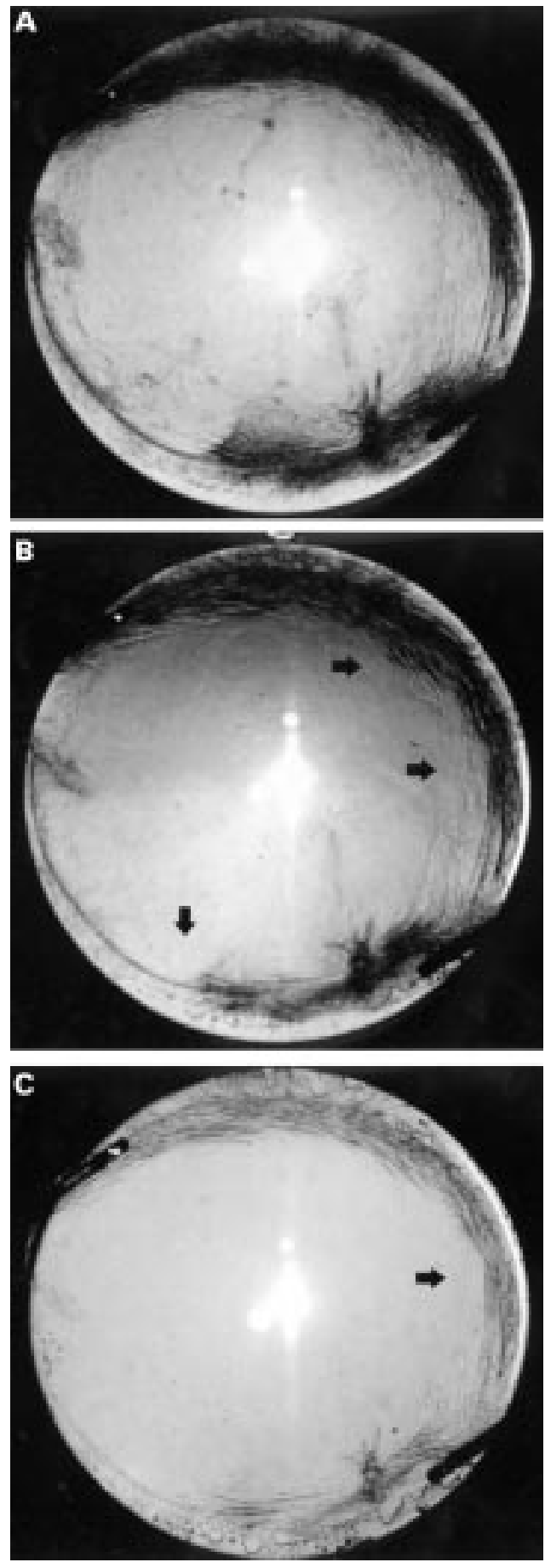

Figure 2 LEC regression with a PMMA len photographed 3 months, 6 months, and 2 years postoperatively. (A) The capsulorhexis is eccentric, lying on the anterior surface of the implant inferiorly, but is retracted off the superior aspect implant to lie on the side of the posterior capsule, forming a dense band of fibrosis. (B) and (C) Minor areas of lens epithelial cell regression can be seen between 6 months and 2 years postoperatively (arrows).

was no statistically significant difference between the PMMA and silicone groups $(p=0.33)$. Of the patients in the polyacrylic group with LECs at day 90 none showed LEC progression, $17 \%$ remained stable, and $83 \%$ demonstrated marked LEC regression (Figs 5A, B, C and 6A, B, C). Delayed LEC migration onto the posterior capsule was seen at 2 years in $16 \%$ patients 

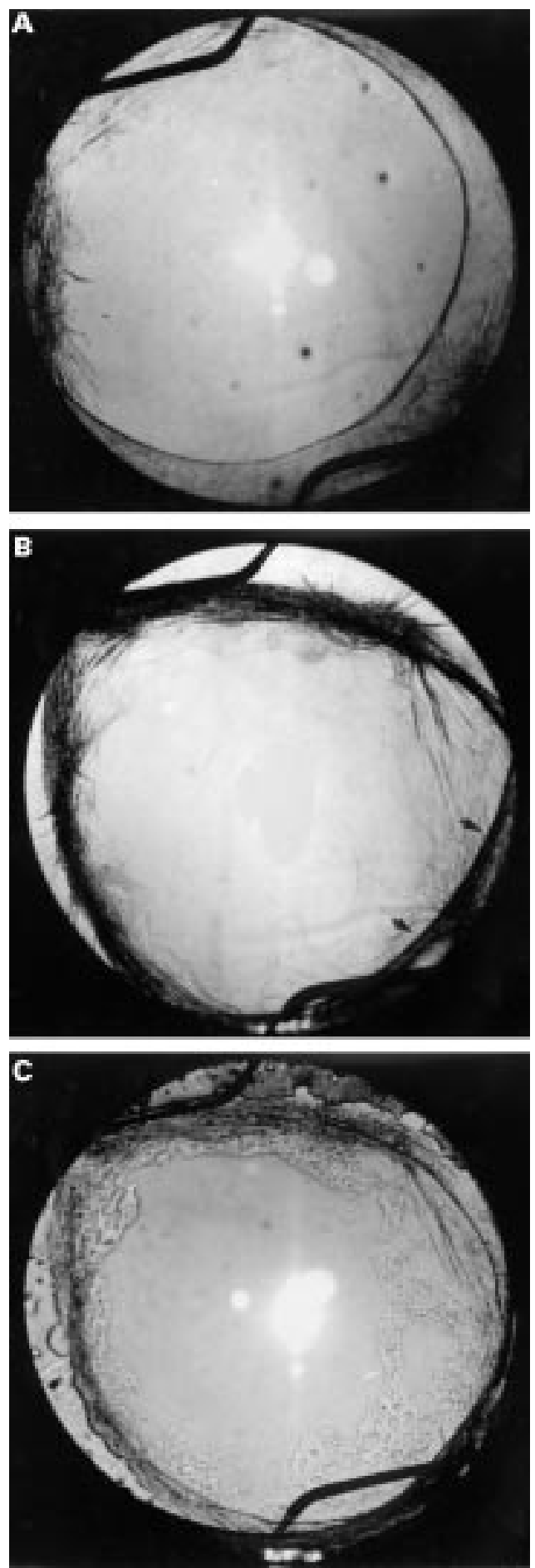

Figure 3 LEC progression with a silicone lens photographed at 3 months, 6 months, and 2 years postoperatively. (A) Three months postoperatively, the capsulorhexis is eccentrically placed on the implant. (B) At 6 months it has retracted off most of the anterior surface of the implant except for a small area laterally (arrows) and set up the formation of dense fibrotic bands where it comes into contact with the posterior capsule (the haptics remain in the bag). (C) At 2 years an LEC membrane can be seen around the periphery although the visual axis remains clear.

with polyacrylic IOLs who had no LECs at 90 days. There is a statistically significant relation between lens type and whether the LECs in the posterior capsule regress, remain stable, or progress $(\mathrm{p}<0.0001)$, with more progression
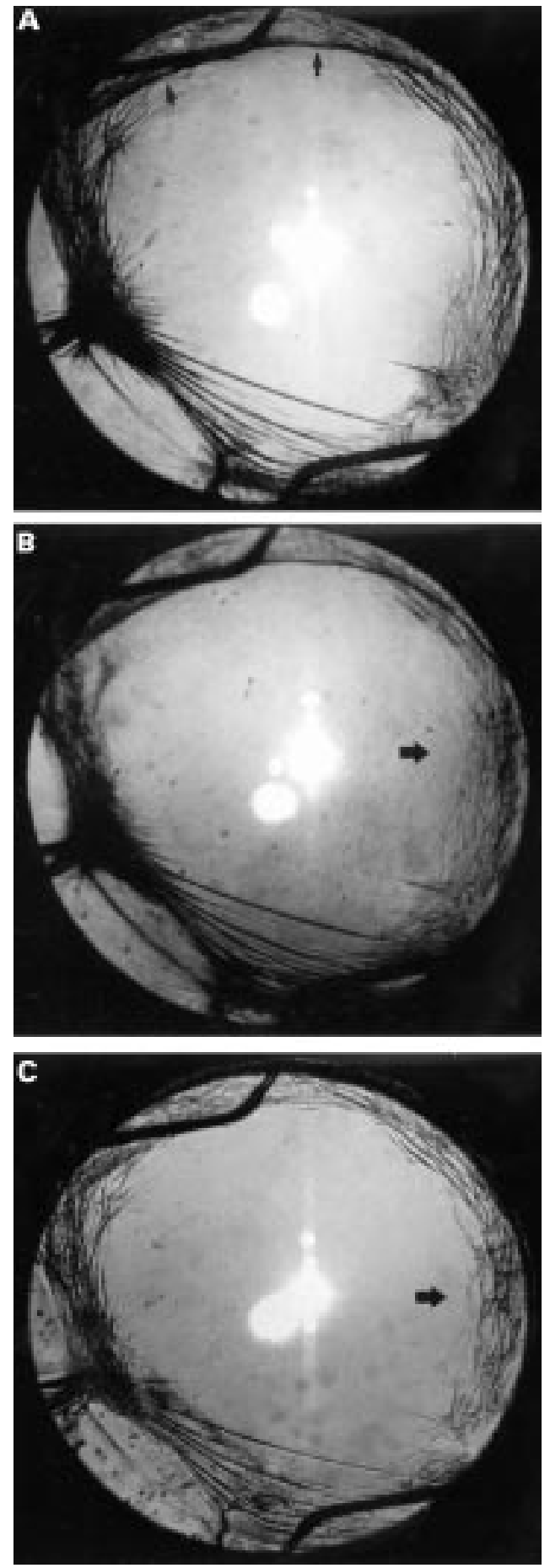

Figure 4 LEC regression with a silicone lens photographed at 3 months, 6 months and 2 years. (A) Three months after surgery the capsulorhexis lies on the anterior surface of the implant superiorly (arrows) but has retracted off the implant elsewhere coming into contact with the posterior capsule and forming a dense fibrotic band. (B) Six months after surgery LEC infiltration is seen on the posterior capsule (arrow). (C) Two years after surgery there has been regression of the LECs in this area (arrow).

being seen with PMMA and silicone and regression with polyacrylic.

A higher number of patients in the polyacrylic group did not attend for follow up at 2 years in comparison with those in the PMMA and silicone groups; this may have been because the patients with polyacrylic IOLs had less PCO and better vision. 

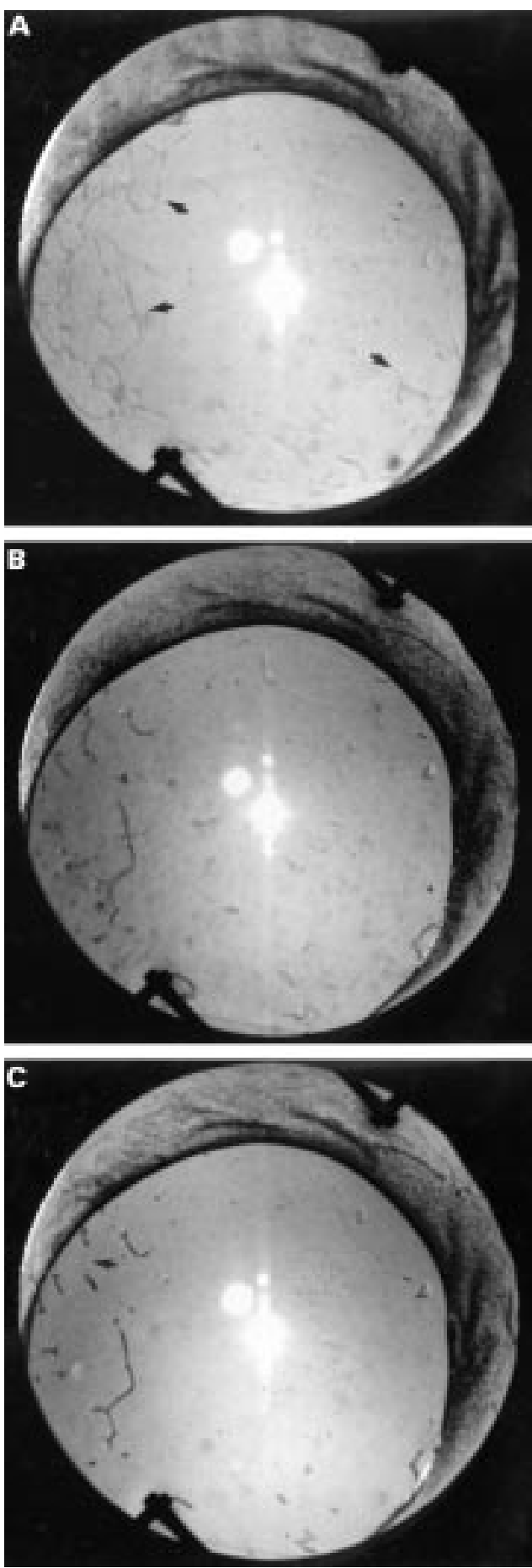

Figure 5 LEC regression with a polyacrylic lens photographed at 1, 3, and 6 months postoperatively. (A) One month after surgery the capsulorhexis is eccentrically placed on the anterior IOL surface. A fine reticular lens epithelial cell network can be seen on the posterior capsule (arrows). (B) Three months after surgery there has been marked regression of these LECs. (C) This LEC regression continues 6 months postoperatively (arrow). The patient failed to attend for further follow up at 2 years.

CAPSULORHEXIS MARGIN CONTACT

The correlation of capsulorhexis contact with the IOL optic for each lens type with the presence of LECs at day 90 is shown in Table 3. It can be seen for all lens materials that if the capsulorhexis edge is completely on the IOL optic the likelihood of LECs growing onto the
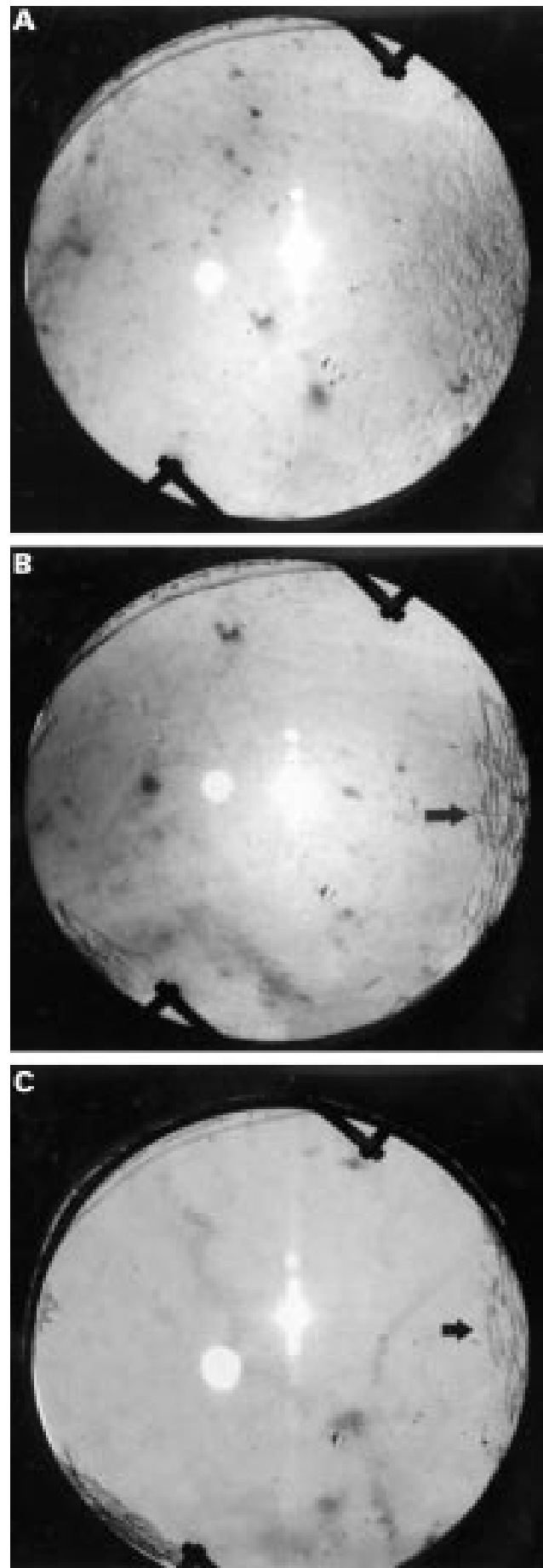

Figure 6 LEC regression with a polyacrylic lens photographed at 1 month, 3 months, and 2 years. (A) The capsulorhexis is only in contact with the implant surface for a small area superiorly. LEC infiltration can be seen in the posterior capsule at 1 month. (B) At 3 months there has been a marked regression of LECs (arrow). (C) This area has regressed further at 2 years postoperatively (arrow).

posterior capsule at 90 days is less than if the capsulorhexis is partly or completely off the optic $(\mathrm{p}<0.0001)$. There was no statistically significant association between lens type and capsulorhexis position $(\mathrm{p}=2.3)$. Consequently the decreased LEC growth seen with polyacrylic lenses cannot be explained by a difference in capsulorhexis position. 
Table 3 Relation of the capsulorhexis contact to the IOL optic and the presence of LECs at 90 days, for each IOL type

\begin{tabular}{|c|c|c|c|c|c|c|}
\hline & \multicolumn{2}{|c|}{$\begin{array}{l}\text { Rhexis completely on } \\
(n=20)\end{array}$} & \multicolumn{2}{|c|}{$\begin{array}{l}\text { Rhexis partly off } \\
(n=53)\end{array}$} & \multicolumn{2}{|c|}{$\begin{array}{l}\text { Rhexis completely off } \\
(n=8)\end{array}$} \\
\hline & No LEC & $L E C$ & No LEC & $L E C$ & No LEC & $L E C$ \\
\hline PMMA $(\mathrm{n}=28)$ & 1 & 5 & 0 & 19 & 0 & 3 \\
\hline Silicone $(n=27)$ & 2 & 3 & 0 & 20 & 0 & 2 \\
\hline Polyacrylic $(n=26)$ & 9 & 0 & 5 & 9 & 0 & 3 \\
\hline Total $(n=81)$ & $12(60 \%)$ & $8(40 \%)$ & $5(9 \%)$ & $48(91 \%)$ & $0(0 \%)$ & $8(100 \%)$ \\
\hline
\end{tabular}

\section{Discussion}

This study shows that the material from which an IOL is made influences whether lens epithelial cells are present on the posterior capsule after cataract surgery and can affect the behaviour of these cells. The presence of LECs on the posterior capsule at 90 days and 2 years was considerably lower with polyacrylic lenses than PMMA or silicone $(\mathrm{p}<0.001)$. Polyacrylic IOLs have been reported to be associated with a low incidence of $\mathrm{PCO}^{17} 18$ and the observation that the cells causing PCO are significantly less likely to be present on the posterior capsule with this lens supports these studies.

We find that in all eyes, regardless of implant material, LEC migration onto the posterior capsule starts within days of surgery so that patterns of LECs are easily identifiable when present on the posterior capsule at 90 days postoperatively. In eyes with PMMA or silicone IOLs this process is usually progressive with only minor degrees of LEC regression being found many months later. In contrast, eyes with polyacrylic IOLs which had LECs at 90 days showed substantial LEC regression between 30 and 90 days postoperatively which then stabilises or continues to slowly regress further with time (Figs 5 and 6). If LECs were present at 90 days regression occurred significantly more frequently with polyacrylic lenses than PMMA or silicone $(\mathrm{p}<0.0001)$. Conversely, LECs are significantly more likely to progress with PMMA and silicone lenses than polyacrylic $(\mathrm{p}<0.0001)$.

The mechanism by which IOL material influences LEC behaviour is unknown but could be explained by either mechanical or material related effects. The higher refractive index of polyacrylic (1.55) compared with silicone (1.41-1.46) and PMMA (1.49) allows these IOLs to have much thinner optics, suggesting that physical bulk cannot be the explanation for the reduced LEC migration. Polyacrylic IOLs have a more defined and squarer optic edge than the other two implants and a recent study has shown a significant decrease in PCO with sharp optic edges. ${ }^{19}$ The authors propose that the sharp edge acts as a mechanical barrier to lens epithelial cell migration onto the posterior capsule. This could be of importance in decreasing the number of cells on the posterior capsules with polyacrylic IOLs but would not explain the phenomenon of LEC regression and the stability of the anterior capsule on the IOL. ${ }^{20}$

Preclinical studies on AcrySof showed no evidence that it was toxic to LECs. ${ }^{21}$ Macrophages can readily be seen on the anterior sur- face of some IOLs postoperatively, particularly in diabetic or uveitic patients, and are thought to be an indication of a foreign body reaction. ${ }^{22}$ In our study no macrophages were seen when LEC regression occurs, suggesting that regression was not a necrotic process.

IOLs made of this polyacrylic have a tacky surface. Indirect evidence for an adhesive relation between polyacrylic IOLs and the capsule has been provided by measuring anterior capsular movement on the lens optic. We have shown that the anterior capsule is much more stable on the anterior surface of a polyacrylic lens than PMMA or silicone and only minor capsule movement occurs with time, possibly as it is stuck to the anterior IOL surface. ${ }^{20}$ Furthermore there are a number of anecdotal accounts from surgeons which suggest that if polyacrylic IOLs have to be explanted for anisometropia they become adherent to the capsule soon after surgery and are relatively difficult to explant in comparison with PMMA or silicone lenses ( $T$ Neuhann, Foldable IOLs: different materials and different designs. Presentation at the XIVth Congress of the European Society of Cataract and Refractive Surgeons, Gothenburg, Sweden, 1996).

This postulated bioadhesion between the capsule and the IOL would mean that there is no space for the LECs to migrate in between the IOL and the posterior capsule. This is analogous to the "no space, no cells" theory which is supported by histological studies on other IOL types showing that cell migration behind the optic can be prevented by a posterior convex or biconvex optic if it is in contact with the adjacent posterior capsule. ${ }^{67}$ A tight adhesive relation between the polyacrylic IOL and the capsular bag could exert compression on the LECs that were present, causing them to regress and atrophy. Alternatively, this adhesion might restrict the access of nutrients and growth factors to the migrated LECs resulting in their regression. Our studies would suggest that the adhesion between a polyacrylic IOL and the capsule takes some weeks to establish during which time some LEC migration can occur, and that the explanation is likely to be due to a material rather than a mechanical effect of the lens implant.

The ideal diameter of the capsulorhexis has yet to be elucidated. Many surgeons believe that the LECs at the equator of the capsular bag are the most important in the pathogenesis of PCO and postulate that a capsulorhexis diameter larger than the IOL optic allows fusion of the anterior capsular flap to the posterior capsule setting up a mechanical barrier to LEC migration from the equator. ${ }^{2324} \mathrm{An}$ alternative view is that LECs originating from the anterior capsule are important in the production of $\mathrm{PCO} .^{2} \mathrm{~A}$ retrospective study on 107 patients who had undergone capsulorhexis and extracapsular cataract surgery with in the bag IOL implantation suggested that capsular opacification was less in the group where the anterior capsule was on the optic for 360 degrees. ${ }^{25}$ The large capsulorhexis in our study meant that nearly all were eccentricity placed, 
with the IOL optic edge exposed in some quadrants. Our results suggest that in all IOL types more LECs migrate behind the IOL optic if the capsulorhexis edge is partly or completely off the IOL optic, compared with when the capsulorhexis edge is on the optic for 360 degrees $(p<0.0001)$.

The observation that LECs are found less frequently on the posterior capsule and are more likely to regress with polyacrylic lenses corroborates the clinical observation of less posterior capsular opacification in these patients ${ }^{17} 18$ and this has important clinical implications for implant surgery.

We thank K Tilling of the Department of Public Health, United and Dental Medical Schools, London, for statistical advice. This study was supported by the Iris Fund for the Prevention of This study was supported by the Iris Fund for the Prevention of
Blindness and an unrestricted research grant from Alcon Laboratories.

The authors have no proprietary interest in any of the materials or equipment discussed in this study.

Presented at ASCRS Boston 1997. Awarded best paper of session.

1 Kappelhof JP, Vrensen GFJM. The pathology of aftercataract. A minireview. Acta Ophthalmol 1992;Suppl 205:13-24

2 McDonnell PJ, Zarbin MA, Green WR. Posterior capsule opacification in pseudophakic eyes. Ophthalmology 1983; 90:1548-53.

3 McDonnell PJ, Roen SL, Glaser BM, et al. Posterior capsule opacification: an in vitro model. Arch Ophthalmol 1985;103 1378-81.

4 McDonnell PJ, Stark W, Green WR. Posterior capsule opacification: a specular microscopic study. Ophthalmology 1984;91:853-6.

5 Martin RG, Sanders DR, Souchek J, et al. Effect of posterior chamber IOL design and surgical placement upon postoperative outcome. F Cataract Refract Surg 1992;18:333-41.

6 erative outcome. F Cataract Refract Surg 1992;18:333-41. lar opacification and intraocular lens decentration. Part 1 . Comparison of various posterior chamber lens designs Comparison of various posterior chamber lens designs
implanted in the rabbit model. F Cataract Refract Surg implanted in the

7 Apple DJ, Solomon KD, Tetz MR, et al. Posterior capsule opacification. Surv Ophthalmol 1992;37:73-116.

8 Cummings JS. Postoperative complications and uncorrected acuities after implantation of plate haptic silicone and three-piece silicone intraocular lenses. $\mathcal{F}$ Cataract Refract Surg 1993;19:263-74.
9 Khalifa MA. Polishing the posterior capsule after extracapThalifa MA. Polishing the posterior capsule after extracapSurg 1992;18:170-3.

10 Nishi O, Nishi K, Harida M. Removal of lens epithelial cells by dispersion with enzymatic treatment following aspiration. Ophthalmic Surg 1991;22:444-50.

11 Nishi O, Nishi K, Harida M. Removal of lens epithelial cells following loosening of the junctional complexes. 7 Cataract Refract Surg 1993;19:56-61.

12 Power WJ, Neylan D, Collum LMT. Daunomycin as an inhibitor of human lens epithelial cell proliferation in culture. F Cataract Refract Surg 1994;20:287-90.

13 Goins KM, Ortiz JR, Fulcher SFA, et al. Inhibition of proliferating lens epithelium with antitransferrin receptor immunotoxin. 7 Cataract Refract Surg 1994;20:513-16.

$14 \mathrm{McD}$ nnell P, Krause W, Glaser B. In vitro inhibition of lens epithelial cell proliferation and migration. Ophthalmic Surg 1988;19:25-30.

15 Pande MV, Ursell PG, Spalton DJ, et al. High resolution digital imaging of the posterior lens capsule after cataract surgery. 7 Cataract Refract Surg 1997;23:1521-7.

16 Pande M. Continuous curvilinear capsulorhexis (circular) and planned extracapsular cataract extraction-are they compatible? Br f Ophthalmol 1993;77:152-7.

17 Oshika T, Suzuki Y, Kizaki H, et al. Two year study of a soft intraocular lens. F Cataract Refract Surg 1996;22:104-9.

18 Ursell PG, Spalton DJ, Pande MV, et al. The relationship between intraocular lens biomaterials and posterior capsule opacification: a 2 year prospective randomised trial comparing PMMA, silicone and polyacrylic lenses. 7 Cataract Refract Surg 1998;24:(in press).

19 Nagata T, Wantanabe I. Optic sharp edge or convexity: comparison of effects on posterior capsular opacification. fpn $\mathcal{F}$ Ophthalmol 1996;40:397-403.

20 Ursell PG, Spalton DJ, Pande MV. Anterior capsule stability in eyes with intraocular lenses made of poly(methyl methacrylate), silicone and AcrySof. 7 Cataract Refract Surg 1997;23:1532-8.

21 Anderson C, Koch DD, Green G, et al. Alcon AcrySof acrylic intraocular lens. In: Martin RG, Gills JP, Sanders $\mathrm{DR}$, eds. Foldable intraocular lenses. Thorofare, NJ: Slack, 1993:161-77.

22 Ygge J, Wenzel M, Philipson B, et al. Cellular reactions on heparin surface-modified versus regular PMMA lenses during the first postoperative month; a double-masked and randomized study using specular microscopy. Ophthalmology 1990;97:1216-24.

23 Tetz M, Imkamp E, Gwin T, et al. Posterior capsular opacification and intraocular lens decentration. $\mathcal{F}$ Cataract Refract Surg 1988;14:614-23.

24 Liu CSC, Wormstone IM, Duncan G, et al. A study of human lens cell growth in vitro: A model for posterior capsule opacification. Invest Ophthalmol Vis Sci 1996;37:906sule 14.

25 Ravalico G, Tognetto D, Palomba MA, et al. Capsulorhexis size and posterior capsule opacification. $\mathcal{F}$ Cataract Refract Surg 1996;22:98-103. 